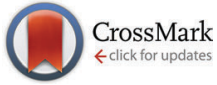

Cite this: J. Mater. Chem. B, 2016, 4, 1528

Received 23rd October 2015, Accepted 25th January 2016

DOI: $10.1039 / c 5 t b 02217 g$

www.rsc.org/MaterialsB

\title{
The influence of selected NSAIDs on volume phase transition in poly(2-(2-methoxyethoxy)ethyl methacrylate) hydrogels $\dagger$
}

\author{
Magdalena N. Olejniczak, ${ }^{\star a}$ Krzysztof Piechocki, ${ }^{a}$ Marcin Kozanecki, ${ }^{a}$ \\ Kaloian Koynov, ${ }^{b}$ Agnieszka Adamus ${ }^{c}$ and Radosław A. Wach ${ }^{c}$
}

\begin{abstract}
Hydrogels exhibiting Volume Phase Transition (VPT) are considered as useful biomaterials for the preparation of various drug delivery systems. Such hydrogels are commonly based on thermo-responsive polymers, such as poly(2-(2-methoxyethoxy)ethyl methacrylate) $\left(\mathrm{PMEO}_{2} \mathrm{MA}\right)$, that have lower critical solution temperature (LCST) in aqueous solutions. In this work, $\mathrm{PMEO}_{2} \mathrm{MA}$ hydrogels were used as model systems to study the influence of encapsulated drugs, such as ibuprofen and salicylate sodium salts, on the temperature and dynamics of the VPT. Both thermo-optical analysis and differential scanning calorimetry have shown that the VPT becomes broader and shifts towards higher temperatures with increasing drug concentration. Three regimes of VPT in $\mathrm{PMEO}_{2} \mathrm{MA}$ gels were distinguished. The first two, related to the breaking of the strong water-polymer interactions and to the network collapse, slow down with increasing drug concentration. The last regime, corresponding to the slow diffusion of a residual solution from a collapsing network, becomes visible only for systems with high content of drug. Raman spectroscopy studies show that the observed effect is not connected to direct interactions between polymers and drugs. This suggests that the drug molecules are able to stabilise water-polymer interactions in thermo-responsive hydrogels. Consequently, they are able to modulate VPT and have a significant influence on the delivery process.
\end{abstract}

\section{Introduction}

High water content makes hydrogels biocompatible and results in a variety of biomedical applications, including contact lenses, soft implants and wound dressings. ${ }^{1}$ Recently, special interest was focused on smart drug delivery systems, such as micelles, vesicles, nanocapsules and nanospheres, dendritic polymers, bioconjugates and hydrogels. Thermo-responsive hydrogels exhibiting Volume Phase Transition (VPT) ${ }^{2}$ seem to be particularly attractive for this purpose because they enable precise control over the dose, release rate and delivery point of drugs. ${ }^{3}$ Increased temperature results in an imbalance between hydrophilic and hydrophobic polymer-water interactions, leading to the collapse of the polymer network and to the abrupt expulsion of liquid content (water or solution). Thus, reaching the VPT temperature $\left(T_{\mathrm{VPT}}\right)$ may trigger the release of a drug that was incorporated

\footnotetext{
${ }^{a}$ Department of Molecular Physics, Faculty of Chemistry,

Lodz University of Technology, Zeromskiego 116, 90-924, Lodz, Poland. E-mail: magdalena.olejniczak@p.lodz.pl

${ }^{b}$ Max Planck Institute for Polymer Research, Ackermannweg 10, D-55021 Mainz, Germany

${ }^{c}$ Institute of Applied Radiation Chemistry, Lodz University of Technology, Wroblewskiego 15, 93-590 Lodz, Poland

$\dagger$ Electronic supplementary information (ESI) available. See DOI: 10.1039/c5tb02217g
}

into a polymer network. The transition temperature, which in turn controls drug release, depends on several factors that may be grouped into two main classes: (i) structure type factors: the ratio and the relative position of the hydrophilic and hydrophobic segments, network topology and tacticity and (ii) content type factors: solvent composition ${ }^{4}$ and the presence of additional solutes. For example, the extensively studied salting-out effect manifests itself in temperature dependent rapid gel collapse at a critical concentration of inorganic salt. It is thought that salts disrupt the hydration structure surrounding the polymer chains, causing a decrease in de-mixing temperature.,

Additionally, aromatic compounds (for example, benzaldehydes) also cause lowering of the lower critical solution temperature (LCST) and $T_{\mathrm{VPT}}$ in a linear polymer solution and gel, respectively. The observed effect depends on the molecular structure and concentration of added organic compounds. Although a clear decrease of LCST was found for all studied systems containing organic compounds, there was no correlation with either solubility or hydrophobicity of the aromatic molecules. ${ }^{7}$

In contrast to the inorganic salts and benzaldehydes, the anionic surfactant sodium dodecyl sulphate (SDS) brings about a large increase of $T_{\mathrm{VPT}}$ or even a disappearance of VPT (salting-in effect). A similar but less pronounced effect was observed also in the case of cationic dodecyltrimethyl ammonium chloride (DTAC). ${ }^{8-10}$ 
Currently, there are two somewhat contradictory explanations of this effect. The first assumes that the surfactants absorb to the polymer chains and substantially disturb the hydrophilic/ hydrophobic balance. ${ }^{9,10}$ The second one proposes a qualitative model for micelle formation inside the gel network. ${ }^{9}$

Investigations addressing the influence of biologically active agents on VPT in stimuli responsive hydrogels are very rare. One of the most essential studies in this field was published by Grinberg et al. ${ }^{11}$ The DSC study of polycationic and polyanionic poly( $N$-isopropylacrylamide) (PNIPAM) copolymer hydrogels showed that the presence of sodium ibuprofen and propranolol hydrochloride caused $T_{\mathrm{VPT}}$ to have lower values in both cases. Additionally, Zuber et al. showed that the presence of bioactive molecules, such as lidocaine and its derivatives (hydrochloride and acetamide), had shifted the LCST of aqueous solutions of the linear copolymer of poly(2-(2-methoxyethoxy)ethyl methacrylate) and poly $(N$-vinylcaprolactam $)$ to lower values. ${ }^{12}$ These data are intriguing in light of the opposite results obtained for $\mathrm{PMEO}_{2} \mathrm{MA}$ hydrogels in the present work.

Most of the aforementioned studies employed systems based on PNIPAM, which is considered to be the "gold standard",13 of thermo-responsive polymers, despite some recent suspicions of its monomer carcinogenicity. ${ }^{14}$ However, the knowledge of the impact of salt (the significant component of body fluids) and the ionic form of biologically active agents (used as drugs due to their good solubility in aqueous systems) on phase transition phenomena in PNIPAM alternatives is particularly important for future applications.

Hydrogels based on thermo-responsive polymers belonging to the poly(oligo(ethylene glycol)methacrylate) (POEGMA) group, such as $\mathrm{PMEO}_{2} \mathrm{MA}$, are distinguishable by the tuneable, fully reversible and sharp VPT with small hysteresis. ${ }^{13}$ A multitude of synthesis methods useful for their preparation (free radical polymerisation - FRP ${ }^{15}$ radiation-induced free radical polymerisation RI-FRP, ${ }^{16}$ atom transfer radical polymerisation - ATRP, ${ }^{17}$ reversible addition-fragmentation chain transfer - RAFT ${ }^{18}$ ) and a variety of feasible structures (bare and decorated networks, brushes, ${ }^{19}$ stars, ${ }^{20}$ nano-, micro- and macrogels ${ }^{21}$ ) are also important advantages of POEGMA materials.

Importantly, the monomer of $\mathrm{PMEO}_{2} \mathrm{MA}$ appears to be nontoxic ${ }^{22}$ and non-immunogenic; ${ }^{23}$ hence, $\mathrm{PMEO}_{2} \mathrm{MA}$ hydrogels constitute promising materials for drug delivery applications.

$T_{\mathrm{VPT}}$ is a critical parameter determining the drug release process; therefore, knowledge of the impact of the drug presence on VPT is highly desirable from both cognitive and practical points of view. In this work, the influence of the presence of non-steroidal anti-inflammatory drugs (NSAIDs) like $(S)$-ibuprofen (IBUNa) and salicylate (SALNa) sodium salts on the $T_{\mathrm{VPT}}$ of $\mathrm{PMEO}_{2} \mathrm{MA}$ hydrogels is analysed within the context of their concentration. Selected drugs are good candidates for model substances due to their relatively simple chemical structure and small size. It is also necessary to underline that simple inorganic salts could not be regarded as a model of bioactive substances (drugs, enzymes, etc.) due to the lack of hydrophobic parts in their chemical structure. Hydrophobic segments may significantly influence the supramolecular structure of water that hydrated them. ${ }^{24}$ The results of thermo-optical analysis (TOA) presented herein are useful to determine a so-called "cloud point", which reflects VPT. These results, supported by differential scanning calorimetry (DSC), allow for the determination of both the temperature and dynamics of this transition. Raman spectroscopy is used to provide more information about the causes of the observed effect at the molecular level.

\section{Materials and methods}

\subsection{Sample preparation}

Simultaneous polymerisation and cross-linking of $\mathrm{PMEO}_{2} \mathrm{MA}$ was attained via electron-beam irradiation of a mixture of 2-(2methoxyethoxy)ethyl methacrylate ( $\left.\mathrm{MEO}_{2} \mathrm{MA}\right), 2$-hydroxyethylmethacrylate (HEMA) and ethylene glycol dimethacrylate (EGDMA) (molar ratios: 100:2:1). ${ }^{16}$ Such composition of substrates and an irradiation dose of $30 \mathrm{kGy}$ were selected to obtain a firm gel with good mechanical properties and, simultaneously, with a high degree of swelling (DS). The irradiation process was carried out at room temperature in $2 \mathrm{ml}$ glass ampoules using the linear accelerator ELU-6 (Eksma, Russia) with a dose rate of ca. $5 \mathrm{kGy} \mathrm{min}^{-1}$. After irradiation, gels were conditioned for 24 hours at $50{ }^{\circ} \mathrm{C}$ to avoid phase separation being a post-polymerisation effect in the utilized system, as we observed previously. ${ }^{16}$

After synthesis, the gels were immersed and equilibrated in deionized water (Merck Millipore Water Purification System) at $7{ }^{\circ} \mathrm{C}$ to extract both sol fraction and unreacted substrates. Water was exchanged every two days until the equilibrium degree of swelling has been reached. Then, the gels were freeze-dried.

\subsection{Drug loading}

Dry samples with a mass of 0.1-0.2 $\mathrm{g}$ were immersed in aqueous solutions of drugs used in significant excess. In the case of $(S)$-ibuprofen sodium salt, the following initial concentrations were used: 5, 10, 20, 30, 41, 100, 140, $182 \mathrm{mM}$, while for salicylate sodium salt: 6.5, 10, 20, 41, 100, $203 \mathrm{mM}$. Gel swelled with $154 \mathrm{mM}$ sodium chloride solution (Baxter, $0.9 \% \mathrm{NaCl}$ solution for infusion) was used as a negative control. Gels were immersed for at least ten days. This time was sufficient to establish thermodynamic equilibrium in the system, as the comparison of the results obtained for gels immersed for 10, 20 and 30 days has not shown differences - see Fig. S1A in the ESI. $\dagger$

Taking into account the potentially different solubility of used drugs in water and in the gel phase, the appropriate partition coefficient was determined as ${ }^{25,26}$

$$
K=\frac{C_{\text {hydrogel }}}{C_{\text {bulk }}}
$$

where $C_{\text {hydrogel }}$ and $C_{\text {bulk }}$ are the drug concentrations in the equilibrium state inside the gel and in the external aqueous solution, respectively. Initial drug concentration $\left(C_{0}\right)$ and $C_{\text {bulk }}$ were spectrophotometrically estimated $\left(\lambda^{\text {IBUNa }}\left(\mathrm{H}_{2} \mathrm{O}\right) / \mathrm{nm} 264\right.$ and $\lambda^{\mathrm{SALNa}}\left(\mathrm{H}_{2} \mathrm{O}\right) / \mathrm{nm} 295$, Carry 5000 Varian Inc.). $C_{\text {hydrogel was }}$ calculated based on the difference between initial $\left(C_{0}\right)$ and final $\left(C_{\text {bulk }}\right)$ drug concentrations in the water phase. 


\subsection{Gravimetric analysis}

The degree of swelling at the equilibrium state $\left(\mathrm{DS}_{\mathrm{eq}}^{\text {water }}\right)$ in water, as well as in the drug solution ( $\left.\mathrm{DS}_{\mathrm{eq}}^{\mathrm{drug}}\right)$, has been calculated as follows:

$$
\mathrm{DS}_{\mathrm{eq}}^{\mathrm{i}}=\frac{m_{\mathrm{s}}-m_{\mathrm{d}}}{m_{\mathrm{d}}}
$$

where $m_{\mathrm{d}}$ and $m_{\mathrm{s}}$ are the dry and swollen gel weights, respectively. The superscript $i$ relates to either water or the drug solution.

\subsection{Thermo-optical analysis}

Thermo-optical analysis (TOA) was performed in the bright field of an optical microscope. The TOA set consisted of a hot stage (Mettler FP 82), a control unit (Mettler FP 90) and a home-made cooling system (presented in Fig. S3 - see ESI $\dagger$ ). In this method, the intensity of white light transmitted through the sample $(I)$ is measured as a function of temperature, and analogous to nephelometry, the cloud point that accompanies VPT may be determined. ${ }^{27}$ Because TOA is a dynamic method (i.e., results are dependent on the scan rate), at first, the heating rate was optimised. Measurements were carried out for a neat $\mathrm{PMEO}_{2} \mathrm{MA}$ hydrogel at various heating rates (see Fig. S2 in the ESI $\dagger$ ). The results acquired at heating rates that varied in the range of 0.5 to $2{ }^{\circ} \mathrm{C} \min ^{-1}$ are practically identical. Only the TOA curve recorded for faster heating $\left(3{ }^{\circ} \mathrm{C} \mathrm{min}^{-1}\right)$ differed from the others. Thus, for further investigations, a $2{ }^{\circ} \mathrm{C} \mathrm{min}^{-1}$ heating rate was chosen because it is close enough to thermodynamic equilibrium and simultaneously allowed for minimising the time of measurement.

Raw data were normalized according to the formula:

$$
I_{\mathrm{N}}(T)=\frac{I_{0}-I_{\mathrm{T}}}{I_{0}-I_{\infty}}
$$

where $I_{\mathrm{N}}$ is the normalised white light intensity and the $0, \infty$ and $T$ indexes relate to starting, final and current temperatures, respectively.

The experiments were carried out in triplicate and were fully repeatable (see Fig. S1B, ESI $\dagger$ ). Presented cloud point temperatures are arithmetic averaged with standard deviation no greater than $0.8{ }^{\circ} \mathrm{C}$.

\subsection{Differential scanning calorimetry}

Alternatively, measurements of VPT were conducted using a DSC technique. The DSC instrument (Q200 TA Instruments) was calibrated for both temperature and heat flow using, respectively, indium (melting temperature of $157^{\circ} \mathrm{C}$ ) and the synthetic sapphire standard with a well characterized specific heat capacity. Hydrogel samples with known mass in the range of 3 to $5 \mathrm{mg}$ were sealed in hermetic aluminium pans and analysed in the temperature range between $0{ }^{\circ} \mathrm{C}$ and $c a .90{ }^{\circ} \mathrm{C}$ at a heating rate of $2^{\circ} \mathrm{C} \mathrm{min}{ }^{-1}$ under a nitrogen flow of $50 \mathrm{ml} \mathrm{min}{ }^{-1}$. Measurements were taken at least twice for all samples. Results were fully reproducible.

\subsection{Raman spectroscopy}

The Raman spectra of drugs, drug solutions and hydrogels were recorded with the use of the FT Raman spectrometer MultiRAM
(Bruker $\mathrm{GmbH}$ ). All of the spectra were collected with a spectral resolution of $4 \mathrm{~cm}^{-1}$ and an excitation wavelength of $1064 \mathrm{~nm}$ (diode Nd:YAG laser, $100 \mathrm{~mW}$ ). During the measurements, hydrogel samples were placed into specially designed cells, guaranteeing a fixed position relative to the laser beam and protecting against water loss. To obtain the high quality spectra required, 32 scans were averaged. Because of the hydrogel thermo-responsivity, all operations and measurements were carried out in an air-conditioned room at $17{ }^{\circ} \mathrm{C}$.

\section{Results and discussion}

Fig. 1 presents selected TOA results obtained for $\mathrm{PMEO}_{2} \mathrm{MA}$ hydrogels immersed in aqueous solutions of IBUNa with various concentrations. It is clear that the "cloud point" (assumed to be represented by the deflection point of the TOA curve) shifts toward higher temperatures as the drug concentration increases. Surprisingly, the concentration dependence of the "cloud point" was found to be linear in a broad range of IBUNa content, up to $180 \mathrm{mM}$ - as presented in Fig. 2. The TOA results are remarkably consistent with DSC data, as shown in Fig. 2, providing the $T_{\mathrm{VPT}}$ directly. A similar tendency is also observed for another tested drug, i.e., SALNa, in contrast to a saline solution, which causes lowering of $T_{\mathrm{VPT}}$ to $14{ }^{\circ} \mathrm{C}$ at a physiological concentration of $\mathrm{NaCl}$ (see Fig. S4 in the ESI $\dagger$ ), as was expected based on literature reports. ${ }^{5,6}$ One can assume that this effect is correlated with a change in $\mathrm{pH}$ of sodium salt solutions. Alvarez-Lorenzo et al. ${ }^{28}$ found that $T_{\mathrm{VPT}}$ slightly increased (from 32 to $34{ }^{\circ} \mathrm{C}$ ) as $\mathrm{pH}$ decreased (from 8 to 3 ) in PNIPAM/chitosan interpenetrated networks. This effect was explained by the ionisation of the amino groups of chitosan chains in acidic medium. However, $\mathrm{pH}$ is an important factor in the case of polyelectrolytes, in which it is useless to explain the observed increase in $T_{\mathrm{VPT}}$ for non-electrolyte $\mathrm{PMEO}_{2} \mathrm{MA}$ because $\mathrm{PMEO}_{2} \mathrm{MA}$ gels are pH-independent, as was previously reported by Paris. ${ }^{29}$ Moreover, performed tests showed that the $\mathrm{pH}$ only slightly varies (in the range of 7 to 8) in investigated $\mathrm{PMEO}_{2} \mathrm{MA}$ gels immersed in IBUNa solutions. Due to the same reason, the mechanism proposed by Tanaka et al. for poly( $\mathrm{N}$-isopropylacrylamide-co-acrylic acid) should be rejected. ${ }^{30}$

In non-ionic polymer networks such as $\mathrm{PMEO}_{2} \mathrm{MA}$ or poly(vinyl methyl methacrylate) (PVME), the polymer-polymer and waterpolymer hydrophobic interactions, as well as the water-polymer and water-water H-bonds, play a key role. ${ }^{31}$

Thus, the most probable explanation is that the drug molecules interact inside the gel similar to surfactants. Two scenarios, which will be considered below, may be proposed in such a case: ${ }^{32}$

- drug molecules are adsorbed onto the polymer chain, directly disturbing the polymer-water interactions, and/or above critical micelle concentration ( $\mathrm{cmc}$ ) the drug molecules self-associate causing the polymer chains to coil to wrap the micelles, ${ }^{33}$

- drug molecules change the supramolecular structure of water (rearrangement of water-water H-bonds) and, as a result, this leads to different polymer chain stability in the system. 
A $\square 5 \mathrm{mM} \circ 30 \mathrm{mM} \nabla 100 \mathrm{mM} \times 140 \mathrm{mM}$

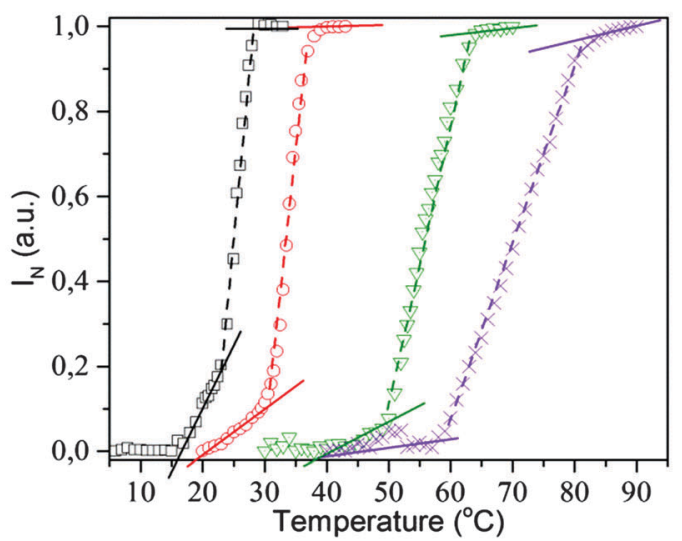

B

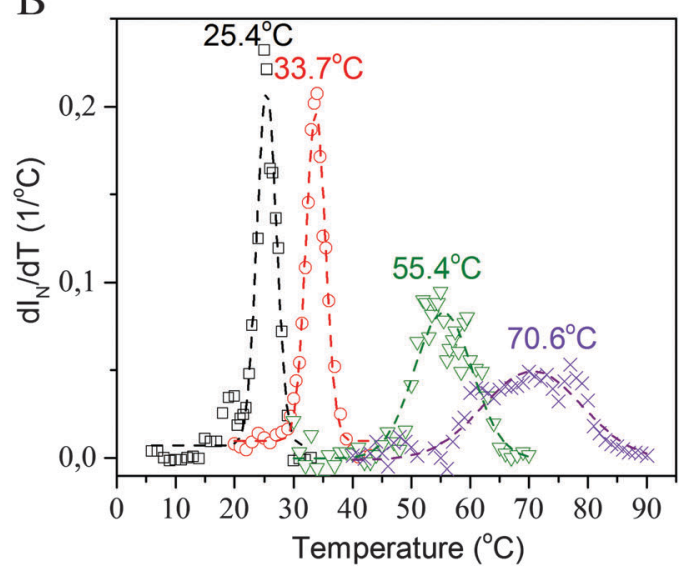

Fig. 1 Influence of IBUNa on VPT in $\mathrm{PMEO}_{2} \mathrm{MA}$ hydrogels. (A) $/_{\mathrm{N}}$ (normalised white light intensity transmitted through the sample) as a function of temperature for $\mathrm{PMEO}_{2} \mathrm{MA}$ hydrogels immersed in aqueous solutions of IBUNa with different concentrations; (B) first derivative of $I_{N}(T)$ with respect to $T$.

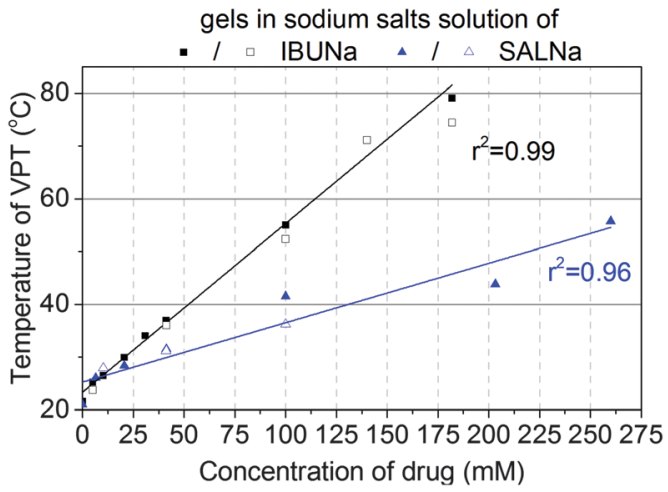

Fig. 2 Comparison of $T_{\text {VPT }}$ determined by DSC and TOA. Dependence of $T_{\text {VPT }}$ as a function of IBUNa concentration - full symbols correspond to the deflection points of TOA curves, while empty symbols correspond to the DSC thermogram extremes. $T_{V P T}$ for SALNa was added for comparison.

Raman spectroscopy is one of the most powerful methods used to test intermolecular interactions in water systems. In the case of hydrogels, it allows for determining the degree of chain hydration and its changes during various transitions, the water structure and even the activation energy of VPT. ${ }^{31,34,35}$
Fig. 3 presents the Raman spectra of IBUNa powder and its aqueous solution in a fingerprint region, divided into three parts for clarity. The slight shift of particular bands toward higher wavenumbers results from hydration of the IBUNa molecules in dissolved form. To control the state of IBUNa in hydrogel, the differential Raman spectrum was calculated by extracting the spectrum of $\mathrm{PMEO}_{2} \mathrm{MA}$ hydrogel swollen in water from the spectrum of $\mathrm{PMEO}_{2} \mathrm{MA}$ hydrogel swollen in IBUNa solution. It was found that the positions of Raman peaks of IBUNa are exactly the same in the hydrogel and in the water solution. This means that there are no specific interactions between drug molecules and polymer chains. IBUNa molecules remain fully hydrated. Thus, the hypothesis that the IBUNa molecules are adsorbed onto the polymer network may be rejected.

The molecular structures of investigated drugs are similar to surfactant molecules with a hydrophobic part and a strongly hydrophilic ionic group. Due to these structural features, they are able to form micelles (cmc for IBUNa and SALNa are $180 \mathrm{mM}^{33}$ and $705 \mathrm{mM},{ }^{36}$ respectively). Nevertheless, the range of concentration investigated in this study does not exceed cmcs. Only one gel sample in $180 \mathrm{mM}$ IBUNa solution reaches the cmc, but no change in $T_{\mathrm{VPT}}$ dependence of drug concentration was observed. Taking into consideration the second scenario it is worth noticing that the polymer chains consist of hydrophilic (easily forming H-bonds) and hydrophobic segments which are encased by water cage-like structures. According to Tanford et al., ${ }^{37}$ in such type of systems, water molecules may form highly ordered structures, called ice-bergs, in the vicinity of hydrophobic polymer chains. The ice-bergs, with water molecules arranged similarly to ice Ih, stabilise the polymer chains in a water environment. Taking into account the lack of direct interactions between the drug and the polymer, it is worth comparing drug concentrations in the gel phase with respect to the initial solution. The partition coefficient of IBUNa and SALNa for various samples was estimated. As the results presented in Table 1 show, the coefficient is almost independent of the initial drug concentration over the investigated concentration range, and the average values are $1.49 \pm 0.04$ and $0.99 \pm 0.06$ for IBUNa and SALNa, respectively. A similar tendency was found for another non-ionic PVME gel used as a Triton adsorbent. ${ }^{38}$ It is also important to note that the $\mathrm{DS}_{\mathrm{eq}}^{\mathrm{ibu}}$ to $\mathrm{DS}_{\mathrm{eq}}^{\text {water }}$ ratios are greater than unity. The results discussed herein are also consistent with the results obtained by Alvarez-Lorenzo et al. ${ }^{28}$ for different thermo-responsive polymer systems - PNIPAM/chitosan interpenetrated networks. This effect was explained by the fact that the free amino groups located along the chitosan chain are cationically charged over a wide range of physiological $\mathrm{pHs}^{28}$ Lowe et al. found that hydrophobic ibuprofen (in the form of acid) induced a decrease of the PNIPAM-based microgels" size below the LCST of PNIPAM and sharpened the VPT. ${ }^{12}$ It was also reported for $\mathrm{PMEO}_{2} \mathrm{MA}$ hydrogels ${ }^{29}$ that $\mathrm{DS}_{\mathrm{eq}}$ reached greater values if the gels were swelled in more basic solutions. Ibuprofen sodium salt, used in these studies, is the sodium salt of weak acid, which results in a slightly alkaline medium $(\mathrm{pH}$ varied between 7 and 8). Thus, the salting in effect was observed. Interestingly, ibuprofen acid incorporated into PNIPAM copolymer hydrogels brought about a drastic decrease of its $\operatorname{DS}_{\text {eq }}$ (salting out effect). ${ }^{12}$ 
A

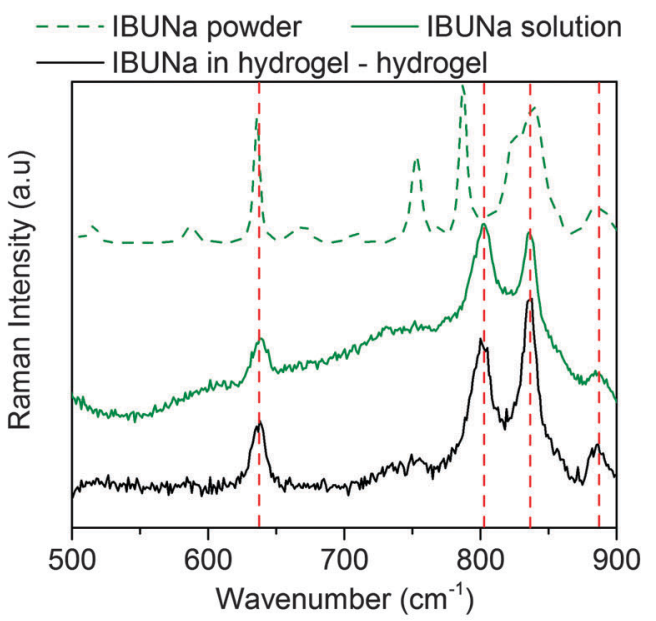

B

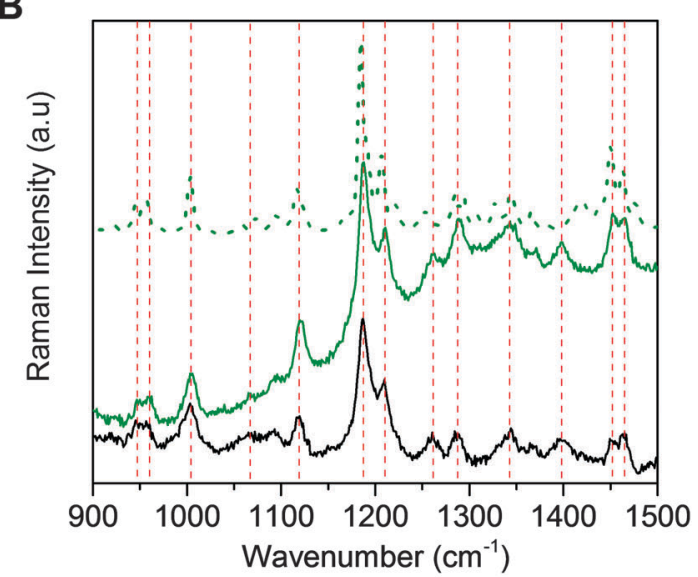

C

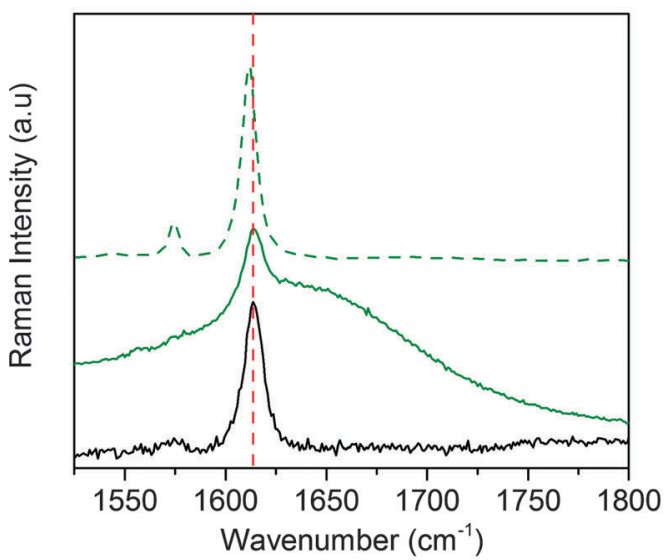

Fig. 3 Raman spectra of IBUNa: powder (green dot line) and aqueous solution (green line) in relation to differential spectrum of IBUNa in hydrogel and hydrogel in three regions: $500-900$ (A), $9000-1500$ (B), $1525-1800 \mathrm{~cm}^{-1}$ (C).

The constant partition coefficient, as well as $\mathrm{DS}_{\mathrm{eq}}^{\mathrm{ibu}}$ to $\mathrm{DS}_{\mathrm{eq}}^{\text {water }}$ ratios, was much greater than unity, which may suggest the formation of a supramolecular structure within the hydrogel, as the simple drug adsorption to the polymer network was excluded by Raman spectroscopy.

The other observed effect relates to the broadening of VPT with increasing drug concentration. This is clearly evident in Fig. 1B, where the temperature dependences of $\mathrm{d} I_{\mathrm{N}} / \mathrm{d} T$
Table 1 Concentration of IBUNa and SALNa: in initial solution ( $C_{0}^{\text {drug}}$ ), in hydrogel $\left(C_{\text {hydrogel }}^{\text {drug }}\right)$ and in the external solution equilibrated with the hydrogel ( $C_{\text {bulk }}^{\text {drug }}$ ); the gel/solution partition coefficient of the drug $(K)$. $\mathrm{DS}_{\text {eq }}^{\text {water }}$ and $\mathrm{DS}_{\mathrm{eq}}^{\text {drug }}$ are degrees of swelling at the equilibrium state in water and in the drug solution, respectively

\begin{tabular}{lclrrr}
\hline $\begin{array}{l}\text { Sodium } \\
\text { salt of }\end{array}$ & $\begin{array}{l}C_{\mathrm{o}}^{\text {drug }} \\
(\mathrm{mM})\end{array}$ & $\mathrm{DS}_{\text {eq }}^{\text {drug }} / \mathrm{DS}_{\text {eq }}^{\text {water }}$ & $\begin{array}{l}C_{\text {hydrogel }}^{\text {drug }} \\
(\mathrm{mM})\end{array}$ & $\begin{array}{l}C_{\text {bulk }}^{\text {drug }} \\
(\mathrm{mM})\end{array}$ & $K$ \\
\hline Ibuprofen & 5 & 1.23 & 6.76 & 4.63 & 1.46 \\
& 10 & 1.89 & 12.76 & 8.51 & 1.50 \\
& 20 & 1.66 & 27.38 & 18.81 & 1.46 \\
& 30 & 1.45 & 43.71 & 28.00 & 1.56 \\
& 75 & 1.68 & 96.00 & 64.29 & 1.50 \\
Salicylate & 6.5 & 0.82 & & & \\
& 10 & 0.89 & 6.34 & 6.73 & 0.94 \\
& 20 & 0.88 & 10.08 & 10.92 & 0.92 \\
& 100 & 0.87 & 20.76 & 19.57 & 1.06 \\
& & & 102.12 & 97.92 & 1.04 \\
& & & & &
\end{tabular}

are presented. This broadening suggests that the VPT becomes continuous in the presence of drug molecules. According to Shibayama and Tanaka, ${ }^{39}$ the discrete or continuous character of the VPT transition relates directly to the temperature dependence of osmotic pressure in the gel. It is easy to imagine that the presence of an ionic substance (drug) in the gel strongly influences this dependence. However, its precise determination is difficult, taking into account all variables changeable during VPT, including the concentration of IBUNa inside the gel, gel volume and intermolecular interactions within the system.

Analysis of the TOA results reveals the presence of three regimes in the VPT. The slopes corresponding to each of them are plotted as a function of IBUNa concentration in Fig. 4 and shown, as straight lines, in Fig. 1A as well. The slopes related to the first and second regimes decrease with IBUNa content. This means that the VPT slows down if the drug concentration increases. The third regime is extremely narrow and practically invisible for solutions with a low concentration of IBUNa, while for higher drug concentrations (above $41 \mathrm{mM}$ ), it becomes visible.

To explain these effects, the comparison of TOA and DSC results was done. Presented in Fig. 5, data for the hydrogel containing $41 \mathrm{mM}$ of IBUNa are representative also for all systems with lower IBUNa content. DSC thermograms show that, although the VPT starts at the same temperature of ca. $25{ }^{\circ} \mathrm{C}$ independent of the drug concentration, the dynamics of the transition are completely different for low and high drug content. Transition is rapid and discontinuous for samples with low drug concentration. This means that most of the waterpolymer interactions break rapidly in the beginning, which is manifested by the sharp energetic effect. Consequently, the hydrogel abruptly becomes thermodynamically instable. The polymer response is also very fast, and rapid collapse of the polymer network leads to an increase of opaqueness manifested by the "cloud point". Higher drug concentration $(\geq 41 \mathrm{mM})$ causes significant redistribution of intermolecular interactions. Strong water-polymer interactions break gradually and disappear completely at higher temperatures. Evidently, IBUNa stabilised the $\mathrm{PMEO}_{2} \mathrm{MA}$-water system. Thus, the hydrogel collapses slowly upon approaching the $T_{\mathrm{VPT}}$ (the first regime in TOA results). The higher the IBUNa content, the slower the process. If a particular number 


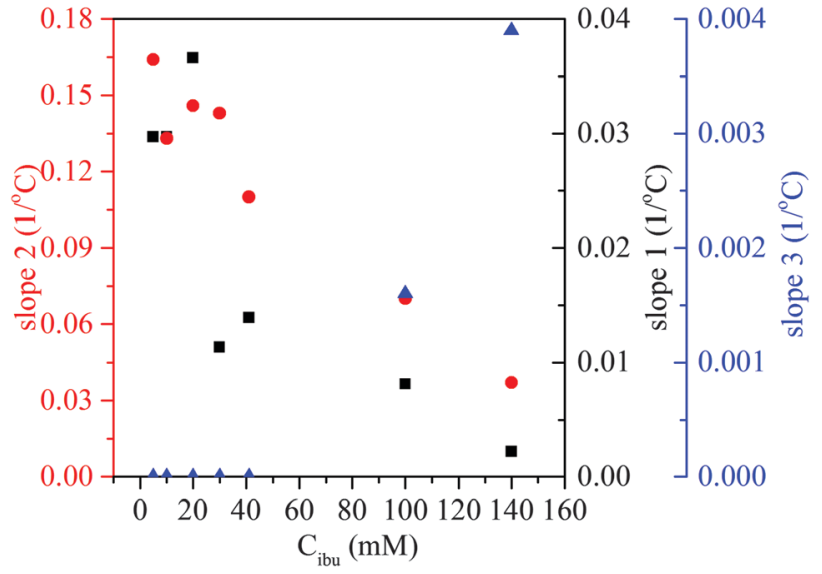

Fig. 4 VPT dynamics in the presence of ibuprofen sodium salt. Dynamics of VPT transition represented by slopes of TOA dependences shown in Fig. 1 in three regimes: slope 1 - squares, slope 2 - dots, slope 3 - triangles (see text for details).

of water-polymer interactions decay, the network becomes unstable. As a result, the gel abruptly reduces its volume. This phase corresponds to the second regime visible in TOA curves and results in the "cloud point". The mentioned herein effects may also be strengthened by the changes in IBUNa concentration inside the gel. It is very probable that the drug molecules, which are much bigger than the water molecules, diffuse outside the gel with a lower rate than the collapse of the gel. As a result, the IBUNa concentration systematically increases with progressing VPT. Thus, the VPT becomes broader for systems with higher drug content. Indeed, micro-Raman spectroscopy investigations performed recently for similar hydrogel systems (based on PVME) containing IBUNa showed that the drug concentration inside the gel may exceed the critical concentration during the VPT, and crystallisation of IBUNa was observed (see Fig. S5 in the ESI $\dagger$ ). Additionally, the presence of the third regime in the TOA experiment, observed for concentrated systems (Fig. 1), supports this hypothesis. The residual amount of IBUNa with strongly limited diffusivity probably protects

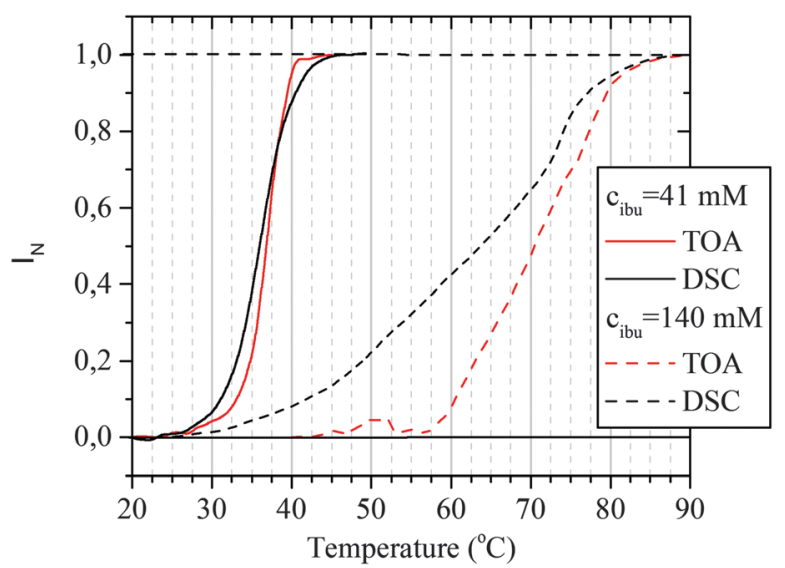

Fig. 5 VPT dynamics in the presence of ibuprofen sodium salt observed using the TOA and DSC methods. Comparison between the DSC thermograms (cumulative representation) and TOA curves measured for two selected $\mathrm{PMEO}_{2} \mathrm{MA}$ hydrogels differed with respect to IBUNa content. the gel against complete collapse. In this phase, the polymer network is partially relaxed and is unable to pull the liquid content out. Contrarily, if the initial drug concentration is low, the internal pressure induced by a rapidly collapsing network is high enough to pull out the whole liquid content. Thus, the third regime is not present for the systems with low IBUNa concentration.

\section{Conclusions}

The influence of the presence of some selected commonly used non-steroidal anti-inflammatory drugs (ibuprofen and salicylate sodium salts) on the VPT in $\mathrm{PMEO}_{2} \mathrm{MA}$ hydrogels synthesised via electron beam irradiation was the subject of the presented investigations. The results of thermo-optical analysis and differential scanning calorimetry showed that the VPT temperature depends on both drug concentration and its chemical structure. Increasing the content of ibuprofen and salicylate sodium salts resulted in a shift of $T_{\mathrm{VPT}}$ towards higher temperatures. Moreover, the VPT became broader. Comparison of TOA and DSC results allowed for distinguishing three regimes of VPT in $\mathrm{PMEO}_{2} \mathrm{MA}$ gels. The first two, related to the breaking of the strong water-polymer interactions and to the network collapse, respectively, slow down with increasing drug concentration. The last regime, corresponding to the slow diffusion of a residual solution from a collapsing network, becomes visible only for systems with high drug content. Such behaviour suggests that the drug molecules stabilised the water-polymer interactions. Comparison of gravimetric and Raman spectroscopy results suggests that the observed effect is due to the stabilisation of water-polymer interactions or water-water structures.

Further investigations are needed to explain the mechanism of the observed effect in detail, however the presented results are indicative of various applications (not only biological). The strong correlation between the $T_{\mathrm{VPT}}$ and drug concentration was demonstrated for $\mathrm{PMEO}_{2} \mathrm{MA}$ but it is probably visible also for other polymer hydrogels. Based on the presented results it may be stated that it is necessary to build a suitable polymer carrier (with particular chemical structure and/or morphology) not only for a particular drug but also for a particular dose.

\section{Acknowledgements}

The authors are deeply grateful to Dr S. Kadlubowski and MSc M. Matusiak for delivery of the hydrogel samples. This work was financially supported by the following projects: 2013/09/B/ ST4/03010 (Polish National Science Centre) and Young Scientists' Fund at the Faculty of Chemistry, Lodz University of Technology (No. W-3/FMN/24G/2015).

\section{References}

1 A. S. Hoffman, Adv. Drug Delivery Rev., 2012, 64, 18.

2 J. Kopeček, Eur. J. Pharm. Sci., 2003, 20, 1.

3 A. Amit, B. Ajazuddin, K. Junaid, S. Swarnlata and S. Shailendra, Eur. J. Pharm. Biopharm., 2014, 88, 575.

4 K. Otake, H. Inomata, M. Konnoa and S. Saito, Macromolecules, 1990, 23, 283. 
5 H. Inomata, S. Goto, K. Otake and S. Saito, Langmuir, 1992, 8, 687.

6 K. van Durme, H. Rahier and B. van Mele, Macromolecules, 2005, 38, 10155.

7 C. Hofmann and M. Schonhoff, Colloid Polym. Sci., 2009, 287(2009), 1369.

8 C. Wu and S. Zhou, J. Polym. Sci., Part B: Polym. Phys., 1996, 34, 1597.

9 H. Inomata, S. Goto and S. Saito, Langmuir, 1992, 8, 1030.

10 D. Schmaljohann, Adv. Drug Delivery Rev., 2006, 58, 1655.

11 V. Y. Grinberg, T. V. Burova, N. V. Grinberg, A. S. Dubovik, A. Concheiro and C. Alvarez-Lorenzo, Langmuir, 2014, 30, 4165.

12 S. Zuber, K. Landfester, D. Crespy and A. Popa, J. Polym. Sci., Part A: Polym. Chem., 2013, 51, 3308.

13 J. F. Lutz, O. Akdemir and A. Hoth, J. Am. Chem. Soc., 2006, 128, 13046.

14 A. S. Wadajkar, B. Koppolu, M. Rahimi and K. T. Nguyen, J. Nanopart. Res., 2009, 11, 1375.

15 Y. Maeda, T. Kubota and H. Yamauchi, Langmuir, 2007, 23, 11259.

16 S. Kadlubowski, M. Matusiak, J. Jenczyk, M. Olejniczak, M. Kozanecki and L. Okrasa, Radiat. Phys. Chem., 2014, 100, 23.

17 J. A. Yoon, T. Kowalewski and K. Matyjaszewski, Macromolecules, 2011, 44, 2261.

18 A. Zengin, Y. Ertan and C. Tuncer, J. Polym. Sci., Part A: Polym. Chem., 2013, 51, 954.

19 S. I. Yamamoto, J. Pietrasik and K. Matyjaszewski, Macromolecules, 2007, 40, 9348.

20 K. Knop, D. Pretzel, A. Urbanek, T. Rudolph, D. H. Scharf, A. Schallon, M. Wagner, S. Schubert, M. Kiehntopf, A. A. Brakhage, F. H. Schacher and U. S. Schubert, Biomacromolecules, 2013, 14, 2536.

21 Z. Hu, T. Cai and C. Chi, Soft Matter, 2010, 6, 2115.

22 H. Vihola, A. Laukkanen, L. Valtola, H. Tenhu and J. Hirnoven, Biomaterials, 2005, 26, 3055.
23 L. Tang, Y. Yang, T. Bai and W. Liu, Biomaterials, 2011, 32, 1943.

24 M. Bester-Rogac, Acta Chim. Slov., 2009, 56, 70.

25 J. Tong and J. l. Anderson, Biophys. J., 1996, 70, 1505.

26 C. Kostmar, T. Sells, N. Taylor, D. E. Liu, J. M. Prausnitz and C. J. Radke, Macromolecules, 2012, 45, 9177.

27 Y. C. Bae, S. M. Lambert, D. S. Soane and J. M. Prausnitz, Macromolecules, 1991, 24, 4403.

28 C. Alvarez-Lorenzo, A. Concheiro, A. S. Dubovik, N. V. Grinberg, T. V. Burova and V. Y. Grinberg, J. Controlled Release, 2005, $102,629$.

29 R. Paris and I. Quijada-Garrido, Eur. Polym. J., 2010, 46, 2156.

30 H. Shunsuke, Y. Hirokawa and T. Tanaka, J. Chem. Phys., 1987, 87, 1392.

31 M. Pastorczak, G. Dominguez-Espinosa, L. Okrasa, M. Pyda, M. Kozanecki, S. Kadlubowski, J. M. Rosiak and J. Ulanski, Colloid Polym. Sci., 2014, 292, 1775.

32 E. Kokufuta, Y.-Q. Zhang, T. Tanaka and A. Mamada, Macromolecules, 1993, 26, 1053.

33 R. Rodríguez, C. Alvarez-Lorenzo and A. Concheiro, Eur. J. Pharm. Sci., 2003, 20, 429.

34 M. Pastorczak, M. Kozanecki and J. Ulanski, Polymer, 2009, 50, 4535.

35 G. E. Walrafen, M. S. Hokmabadi and W.-H. Yang, J. Phys. Chem., 1988, 92, 2433.

36 Y. Tanaka, Y. Miyazaki, S. Yakou and K. Takayama, Pharmazie, 2007, 62, 41.

37 C. Tanford, The Hydrophobic Effect: Formation of Micelles and Biological Membranes, J. Wiley, 2nd edn, 1980.

38 K. Yamagiwa, M. Katoh, M. Yoshida, A. Ohkawa and H. Ichijo, Water Sci. Technol., 1997, 37, 213.

39 M. Shibayama and T. Tanaka, Volume phase transition and related phenomena of polymer gels, Responsive gels: volume transitions I, Springer, Berlin, Heidelberg, 1993, vol. 109, p. 1. 